\title{
Dental hygiene habits and oral health status of seafarers
}

\author{
Syed Sarosh Mahdi ${ }^{1}$, Fabio Sibilio ${ }^{2}$, Francesco Amenta ${ }^{1,2}$ \\ ${ }^{1}$ Telemedicine and Telepharmacy Centre, University of Camerino, Camerino, Italy \\ ${ }^{2}$ Research Department, International Radio Medical Centre (CIRM), Rome, Italy
}

\begin{abstract}
Background: This study has assessed the dental hygiene habits and problems of seafarers and their attitudes/perceptions regarding oral hygiene using a dental hygiene/habits questionnaire.

Materials and methods: A research questionnaire on oral hygiene habits was prepared along with a summary of all the questions and sent to ships via e-mail by Centro Internazionale Radio Medico (CIRM) networks. CIRM, is the Italian Telemedical Maritime Assistance Service (TMAS), and represents the Centre with the largest number of seafarers assisted on board ships worldwide. CIRM proposed the questionnaire to all ships $(n=1,198)$ asking for medical advice from 1 July 2014 till 31 October 2014. Two dental professionals were involved in the development and analysis of the questionnaire.

Results: Seafarers are at risk of several dental health problems due to their oral hygiene and dietary habits, smoking and alcohol consumption, poor oral hygiene knowledge and motivation. Dietary habits during voyages were also questionable and seafarers consume food rich in fermentable carbohydrates, which is a major risk factor for dental caries.

Conclusions: Seafarers need better oral hygiene education and care to enable them to manage their oral health in a better way. Life at the sea, under challenging circumstances is not without stress, that is why it is important that seafarers are given complete information about correct oral hygiene protocols and dental hygiene and the advantages for their health of keeping a healthy mouth.
\end{abstract}

(Int Marit Health 2016; 67, 1: 9-13)

Key words: seafarers, dental hygiene, questionnaire, oral health, prevention

\section{INTRODUCTION}

Dental problems and oral pathologies are not infrequent among seafarers and these workers represent a group of special needs as they are isolated during long sea voyages [1]. On the other hand, provision of health care (including dental care) to sailors is a problematic task, as a large majority of merchant ships do not carry health professionals and are at sea for days or weeks before they can reach a port [2]. For centuries, the captain of the ship has been in charge of the treatment of diseases and the health protection of the crew, while at sea. The possibility of providing medical advice to ships via telecommunication systems became possible with the development of radiotelegraphy by Guglielmo Marconi in 1897. At present, telemedicine is the only mean by which it is possible to get medical advice of a reasonable quality at sea, and there are significant advantages as well as limitations with this approach [3].

Poor oral health of seafarers is considered as a main cause of their dental problems that can result in complications (landing of the patient, diversions from the route) for shipping companies during voyages [1]. Another reason of frequent oral pathologies encountered in sailors is the use of excessive amounts of snacks. They also consume large quantity of tea, coffee and beverages because of their odd working hours and unique lifestyle. Most of dietary substances mentioned above contain fermentable carbohydrates and sugars, which are considered to be prime risk factors of dental caries and associated dental diseases [4, 5]. The

Dr Syed Sarosh Mahdi, School of Life Science (E-Health and Telemedicine), University of Camerino, Via Madonna delle Carceri, 9, 62032 Camerino (MC), Italy,

tel: +39-3662280520, e-mail: syedsarosh.mahdi@unicam.it 
topic of oral health of seafarers has been recently reviewed [1]. This paper has reported the lack of extensive information excluding some basic epidemiological studies among merchant ship crews. This review has also demonstrated that people working on board of navy units in general receive better and articulated dental care [1].

Based on the above consideration Centro Internazionale Radio Medico (CIRM), the Italian Telemedical Maritime Assistance Service (TMAS), developed and distributed a questionnaire on oral health habits of seafarers [6].

\section{MATERIALS AND METHODS}

An anonymous research questionnaire on oral hygiene was prepared [7]. It was proposed that all ships asking for medical advice from CIRM between $1^{\text {st }}$ July 2014 and $31^{\text {st }}$ October 2014 from CIRM should be requested to fill in the questionnaire. The questionnaire was prepared in English and Italian. A summary of all the questions on the questionnaire was also prepared for the captain of each ship to save time and paper work. Informed consent was taken from all sailors who wanted to be part of the research. Those interested in contributing to the initiative were required to send an e-mail in response, after which questionnaires and instructions were sent to the participating ships. In the 4 months CIRM conducted this survey, 1,198 ships required medical assistance to the Centre, but only 65 ships replied to CIRM's request and took part in the study by returning the summary of the filled questionnaires. The demographics of the sample taking part in the survey are shown in Table 1.

The captain of each ship was informed that he can fill the summarised chart after getting responses of all the seamen on board. In case the captain had any queries he was asked to contact via e-mail the CIRM dental specialists in charge of the project. To appreciate the efforts of the captain being part in this survey, CIRM also awarded certificates and appreciation letters. Ships collaborating with the initiative also received a complimentary copy of the book "Oral Hygiene and its Maintenance", specifically developed for increasing the awareness of seafarers to the problem [2].

The data analysis of the questionnaire was done as an active and interactive process. Researchers carefully sifted the results and analysed the data recorded through the questionnaire. Data was analysed after organising the questionnaire results systematically $[8,9]$. Data analysis is the "Process of fitting data together, of making the invisible obvious, of linking and attributing consequences to anteced- ents. This is the process of conjecture and verification, of correction and modification, of suggestion and defence" [10]. At the outset the data were coded to form categories and subcategories emerging from the data. Codes were developed based on the list of themes. Coding as method of conceptualising research data and classifying them into meaningful and relevant categories for the participations in the study [11].

Charts were created and the results were pasted on these charts into different sections and categories and finally typed into Excel files. After the whole process was completed, a list of themes of categories and subcategories were identified and data was compiled and discussed under the identified categories.

\section{RESULTS}

Figure 1 shows the research questionnaire [7]. The results of the survey are summarised in Table 2. A large percentage of seafarers consumed alcohol and nicotine during the voyages. $56.11 \%$ of sailors surveyed $(n=1,156)$ stated that they regularly smoked, out of that population, almost $45 \%$ of the smokers said they smoked more than 10 cigarettes a day. $11.45 \%$ of the total survey population $(n=236)$ responded that they consumed alcohol on a regular basis. In recent decades shipping companies have made an effort to restrict alcohol use on cargo ships but policies and implementation of the policies varies [12]. Cross tabulation between the population that consumed alcohol and smoked tobacco showed that $15.39 \%$ of smokers $(n=178)$ also consumed alcohol. Sailors who consumed alcohol were more likely to smoke $(80.93 \%, n=191)$, sample population that consumed alcohol admitted that they also smoked tobacco.

$55.67 \%$ of the participants ( $n=1,147$ ) acknowledged use of dairy products or candies which contain fermentable carbohydrates. Only the $61.11 \%$ of sailors $(n=1,259)$ had a complete set of natural tooth. $82 \%(n=656)$ of the total 801 sailors without a complete set of teeth, lost their tooth due to extraction. The remaining people lost their tooth as a result of tooth mobility.

Cross tabulation of the data set showed that older sailors (50-60 years) had higher frequency of tooth loss. $6.6 \%$ of the total sample population ( $n=137$ ) was from this age group (50-60 years), $63.5 \%$ of them $(n=87)$ had missing tooth or several missing teeth. Around $30.48 \%$ of the sailors ( $n=628$ ) reported suffering from diseases of the supporting structure of teeth including gingivitis or periodontitis at some

Table 1. Demographics of study sample

\begin{tabular}{llll}
\hline Ships contacted & Took part in survey & No. of seafarers filling in the questionnaire & Rate of return \\
\hline 1,198 & 65 & 2,060 & $5.4 \%$
\end{tabular}




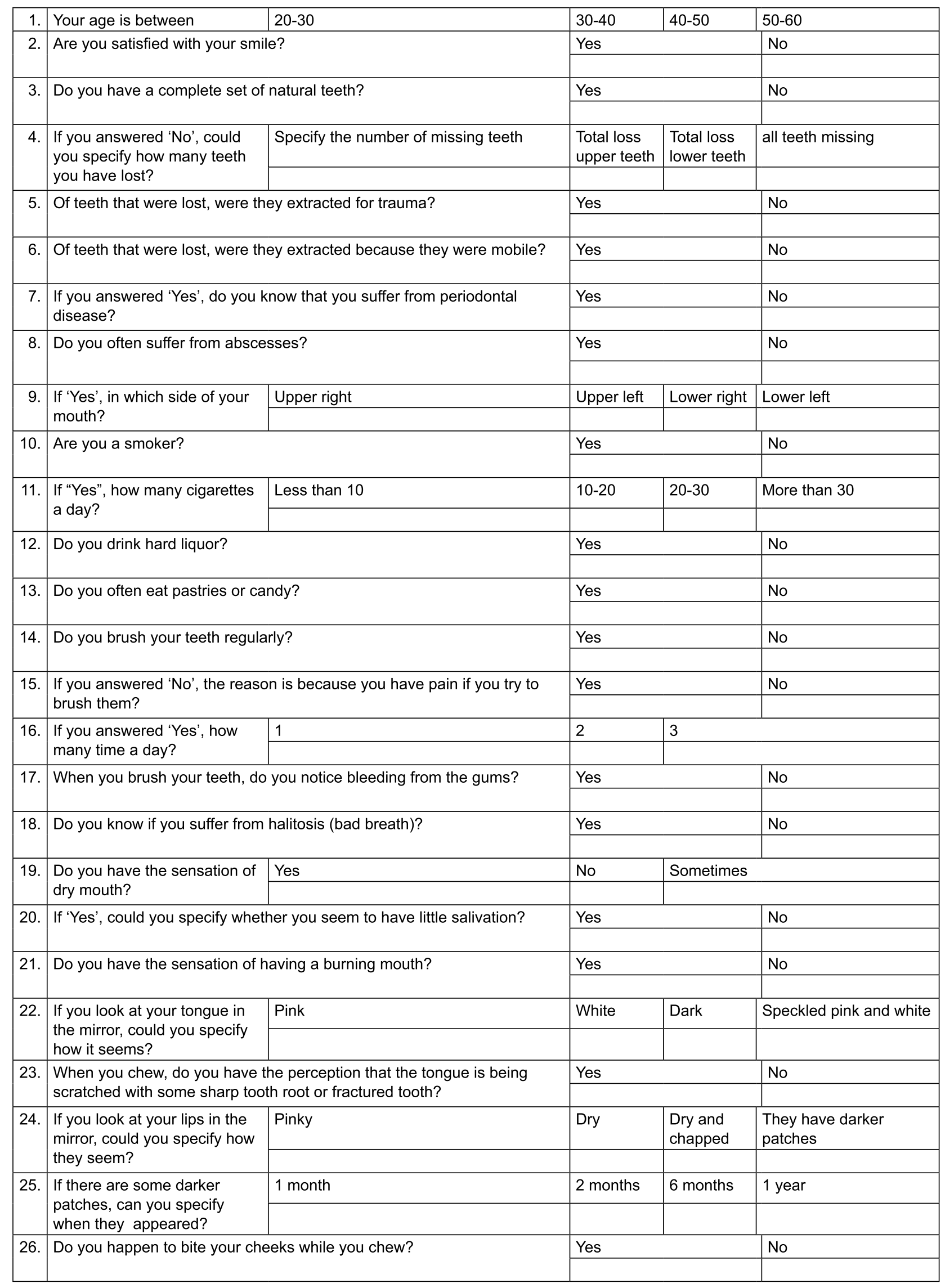

Figure 1. Questionnaire proposed to seafarers on board ships taking part to this survey 
Table 2. Summary of key findings

\begin{tabular}{ll}
\hline \multicolumn{2}{l}{ Synthesis of the key results of the survey questionnaire } \\
\hline Variable & No. (\%) of seafarers \\
\hline Tobacco use & $1,156(56.11 \%)$ \\
Alcohol use & $236(11.45 \%)$ \\
Smokers who also consumed alcohol & $178(15.39 \%)$ \\
Alcohol consumers who also smoked & $191(80.93 \%)$ \\
Regular use of fermentable & $1,147(55.67 \%)$ \\
carbohydrates & \\
Twice a day tooth brushing & $834(40.48 \%)$ \\
Sailors with complete teeth set of teeth & $1,259(61.11 \%)$
\end{tabular}

point of their life. $27.03 \%$ of the sample $(n=557)$ reported bleeding gums during brushing. Over $75 \%$ of the sample were satisfied with their smile.

$74.07 \%$ of the total sample population ( $n=1,526)$ were regularly brushing their teeth, but interestingly only $40.48 \%$ seafarers $(n=834)$ questioned brushed their teeth twice, which is recommended by dental professionals around the world, whereas the majority of them only brushed once a day. $5 \%$ of the sailors surveyed reported that pain was a reason for not brushing their teeth regularly. $4.02 \%$ of the total sample $(n=83)$ reported bad breath or halitosis, whereas only $1.31 \%(n=27)$ sample population indicted feeling the sensation of burning mouth. Whereas $2 \%$ of the sailors also referred occasional cheek biting while chewing or eating.

\section{DISCUSSION}

Dental fitness of seafarers is considered critical by International Labour Organisation (ILO) and World Health Organisation (WHO) [13]. Seafarers are known to disregard oral hygiene during long voyages and also tend to consume high quantity of nicotine, which is probably done to relieve occupational stress due to odd duty hours and isolation [14]. Nicotine is considered the most important preventable risk factor associated with periodontal and lung disease [15]. The Danish Radiomedical service in its annual report 2010, which analysed 1,300 consultations, stated that dental problems (8.1\%) were among the four most reported health issues of seafarers [16]. A study reported that on commercial cargo vessels, the causes of consultations for oral health problems at foreign ports can be as high as 67\% [17]. A survey conducted in 1995 found that only 19 countries provide comprehensive oral health services for seafarers [18]. That is why more research is required to investigate the oral hygiene status of seafarers and to assess their awareness and concerns regarding oral health.
The results of the survey are reflective of poor oral hygiene of seafarers. Seafarers were also found to be smoking more than the general population. Global modelled age-standardised prevalence of daily tobacco smoking in the population older than 15 years was found to be $31 \%$ in 2012 [19], while $48 \%$ of the world population consumes alcohol [20]. In our sample of seafarers, $56 \%$ of them smoked and the $11 \%$ of them consumed alcohol (Table 2). Alcohol consumption on board vessels is a tricky subject as many shipping companies have banned or restricted alcohol consumption altogether, but implementation of these policies remains a subject of debate, hence it is entirely possible that seafarers are hesitant to openly talk about the subject [12]. The higher consumption of tobacco compared to the general population can be attributed to various factors including isolation, boredom and stress. The intake of dairy products and sweets were also deemed to be very high, which is clearly associated with high dental caries risk. Although a majority of the sailors surveyed were brushing their teeth, most of them were only brushing once, perhaps due to inadequate knowledge of oral hygiene protocols. Brushing the teeth twice a day with a fluoridated tooth paste is the recommended practice for maintaining good oral hygiene [21].

Over a quarter of the survey population suffered from bleeding gums which is troubling, and it could also signify underlying systemic diseases. Over half of the population didn't have complete natural teeth set which suggests that seafarers are more likely to lose tooth than the general population, perhaps due to ambivalent attitude towards oral hygiene.

The combination of high intake of alcohol, tobacco, refined carbohydrates (sweets/dairy products) and insufficient knowledge regarding oral hygiene creates a synergistic effect which is evident from the poor oral hygiene status exhibited by a majority of the seafarers. The fact that most seafarers only brushed teeth once daily is also proof enough that oral hygiene knowledge and motivation remains low in this group. There is a dire need to improve the motivation and knowledge level of seafarers regarding oral hygiene practices. Seafarers must also be made aware of the extreme dangers associated with the use of excessive amounts of alcohol, nicotine and refined carbohydrates. Alcohol and smoking are independent risk factors of many diseases, not just limited to diseases of the mouth, including oral cancer and submucous fibrosis [22].

In view of the oral status of seafarers, of the isolation of the ship and of the difficult access to health and dental care of seafarers, telemedicine based applications for dentistry (teledentistry) could represent a way for providing a reasonable level of oral assistance to seafarers. The emergence of teledentistry as a branch of telemedicine can be 
traced back to 1994 when a United States Military project demonstrated that teledentistry reduced dental treatment costs and proved to be effective in providing dental care to distant communities living in rural areas [2]. Teledentistry has the potential to improve access to oral health care and decrease treatment costs [23] and could be integrated with electronic health records, digital imaging as a support of teleconsultations with dental specialists [24]. Teledentistry has been successfully implemented as models to improve dental education and access to care [25] and is effective in the management of oral health issues in remote and rural areas, where access to dentists and oral health specialists is limited [26, 27]. Teledentistry may become a new instrument for tending to the poor oral/dental conditions of people on board of seagoing vessels.

\section{CONCLUSIONS}

The results of this survey have shed light on important dental hygiene issues of seafarers. From the above discussion, it is clear that seafarers need better oral hygiene education and care to enable them to take care of their oral health in a better way. Life at the sea, under challenging circumstances is not without stress, that is why it is important that seafarers are given complete information about correct oral hygiene protocols and dental hygiene. Shipping companies should make sure that seafarers are exposed to regular health examinations including dental checkups, which should be made part of the health fitness test that are conducted before the sailor goes on board. Shipping companies should also develop comprehensive dental hygiene programs for seafarers which have shown to make a profound effect on overall dental health of any community [28]. Seafarers due to their unique lifestyle, represent a vulnerable community as far as oral health is considered, that is why more research is required on dental hygiene habits of sailors to expand our current understanding of the problems this community faces in maintaining its oral health, which has direct implications on overall health.

\section{ACKNOWLEDGEMENTS}

This work was supported by an institutional grant by University of Camerino School of Life Sciences. All authors declare that there is no conflict of interest regarding the publication of this article.

\section{REFERENCES}

1. Sobotta BA, Reiber T, Nitschke I. Oral health of seafarers. A review. Int Marit Health 2011; 62: 8-16.

2. Amenta F, Dauri A, Rizzo A. Organization and activities of the International Radio Medical Centre(CIRM). J Telemed Telecare 1996; 2: 125-131.

3. Goethe WHG. Medical care on ships without a doctor. Radio medical advice. In: Goethe WHG, Watson EN, Jones DT eds. Handbook of Nautical Medicine, Springer, Berlin, 1984, pp. 53-65.
4. Holttinen T, Murtomaa H, Pentti J, Alvesalo I. Utilization of Dental services by Finnish seaman. Acta Odontologica Scandinavia 1994; 52: 71-81.

5. American Dietetic Association. Position paper: nutrition and oral health. J Am Dietary Association 2003; 103: 615-625.

6. International Maritime Organization (IMO). Medical Assistance at Sea. Circular MSC/Circ.960. IMO, London, 2000.

7. Mahdi SS, Mancini M, Sibilio F, Amenta F. Research questionnaire on perception of seafarers about oral hygiene and oral dietary habits. World J Dentistry 2015; 6: 1-4.

8. Patton M. Qualitative evaluation and research methods. Newbury Park, Calif, Sage 1990.

9. Morse JM, Field PA. Qualitative research methods for health professionals. Calif, Sage 1995.

10. Polit D, Beck C. Essentials of nursing research. Wolters Kluwer Health/Lippincott Williams \& Wilkins, Philadelphia 2010.

11. Bowling A. Research Methods in Health: Investigating Health and Health Services Buckingham: Open University Press; 2002.

12. Ames G, Cunradi C, Moore R, Duke M. The Impact of Occupational Culture on Drinking Behavior of Young Adults in the U.S. Navy. J Mixed Methods 2009; 3: 120-150.

13. ILO/WHO. Guidelines for conducting Pre-Sea and Periodic Medical Fitness Examinations for SeaFarers 1997 www.ilo.org/public/ english/dialogue/sector/techmeet/ilowho97/index.htm.

14. Dahl E. Sick leave aboard. A one-year descriptive study among crew on a passenger ship. Int Marit Health 2005; 56: 5-16.

15. Do LG, Spencer AJ, Dost F, Farah CS. Oral mucosal lesions: findings from the Australian National Survey of Adult Oral Health. Australian Dental J 2014; 59: 112-114.

16. Radio Medical Danmark. Annual Report 2010. Esbjerg, 2011.

17. Tomaszunas S. The work of ship's doctors of Polish Ocean Lines. Bull Inst Marit Trop Med Gdynia 1985; 36: 51-58.

18. Saarni U-M, Saarni H, Holttinen T, Fellman MT. Seafarer's dental health: an international problem? In: Third International Symposium on Maritime Health. Maritime Institute of Technology and Graduate Studies, Baltimore, MD, 1995; pp. 139-142.

19. Ng M, Freeman M, Fleming T et al. Smoking Prevalence and Cigarette Consumption in 187 Countries, 1980-2012. JAMA 2014; 311: 183.

20. Anderson P. Global use of alcohol, drugs and tobacco. Drug Alcohol Rev 2006; 25: 489-502.

21. Chester RK, Huntington E, Burchell CK, Stephen KW. Effect of oral care habits on caries in adolescents. Caries Res 1992; 26 : 299-304.

22. Blot WJ, McLaughlin JK, Winn DM. Smoking and drinking in relation to oral and pharyngeal cancer. Cancer Res 1988; 48: 3282-3287.

23. Mihailovic B, Miladinovic M, Vujicic B. Telemedicine in Dentistry (Teledentistry). In: Graschew G, Roelofs TA eds. Advances in Telemedicine: Applications in Various Medical Disciplines and Geographical Areas. InTech, Rijeka (Croatia), 2011;. pp. 215-230.

24. Friction J, Chen $\mathrm{H}$. Using teledentistry to improve access to dental care for the underserved. Dental Clinics of North America 2009; 53: 537-549.

25. Chen J, Hobdell M, Dunn K, Johnson K, Zhang J. Teledentistry and its use in dental education. J Am Dental Association 2003; 134 : 342-346.

26. Yoshinaga L. The use of teledentistry for remote learning applications. Practical Procedures Aesthetic Dentistry 2001; 13: 327-328.

27. Chen H, Fricton J. Teledentistry: seeing the doctor from a distance. Northwest Dentistry 2007; 86: 27-68.

28. Sheiham A. Oral health policy and prevention. In: Murray JJ ed. The prevention of oral disease. 3rd Ed. Oxford University Press, New York, 1996, pp. 234-249. 